\title{
Insuficiencia respiratoria aguda como forma de debut de síndrome de Lambert-Eaton asociado a carcinoma pulmonar de células pequeñas
}

\author{
F. JOVER*, E. CHINER, J. M. ARRIERO, J. SIGNES-COSTA, J. MARCO, \\ V. IZURA** \\ *Servicio de Medicina Interna, Neumología y **Neurofisiología. Hospital Clínico \\ Universitario de San Juan. Alicante
}

\begin{abstract}
LAMBERT-EATON MYASTHENIC SYNDROME PRESENTING AS ACUTE RESPIRATORY FAILURE IN A PATIENT WITH SMALL
\end{abstract} CELL LUNG CANCER

\section{RESUMEN}

El síndrome de Lambert-Eaton (SLE) es un trastorno raro de la transmisión neuromuscular que se presenta habitualmente como un proceso paraneoplásico frecuentemente asociado al carcinoma pulmonar de células pequeñas. Varios estudios han demostrado la existencia de disfunción de la musculatura respiratoria en estos pacientes. La insuficiencia respiratoria aguda como presentación del SLE ha sido descrita de forma excepcional. Se describe un caso de insuficiencia respiratoria aguda como forma de debut de SLE asociado a un carcinoma microcítico de pulmón, repasando las principales características del cuadro y su tratamiento.

El SLE debería ser considerado en casos de insuficiencia respiratoria aguda sin causa aparente y sospecha de enfermedad neoplásica de base. Proponemos la realización de estudios electromiográficos que detecten alteraciones en la función muscular respiratoria, incluyendo el diafragma, para descartar su existencia.

PALABRAS CLAVE: Síndrome de Lambert-Eaton. Insuficiencia respiratoria aguda. Carcinoma pulmonar de células pequeñas.

\begin{abstract}
The Lambert-Eaton myasthenic syndrome is a rare disorder of neu romuscular transmission, usually presenting as a paraneoplastic process associated with a small cell lung cancer. Recently, respiratory muscular impairment has been described in these patients. Acute respiratory failure as a presenting symptom has been reported in few cases. We present a case of acute ventilatory failure as the first manifestation of LambertEaton myasthenic syndrome associated with small cell lung cancer and discuss the main features of this disease, including its treatment.

The Lambert-Eaton myasthenic syndrome should be considered in cases of unexplained acute respiratory failure and clinical evidence of neoplasic disease. We thought that electromyographic studies could reveal the real involvement of respiratory muscles, including diaphragm, in this condition.
\end{abstract}

KEY WORDS: Lambert-Eaton myasthenic syndrome. Acute respiratory failure. Small cell lung cancer.

Jover F, Chiner E, Arriero JM, Signes-Costa J, Marco J, Izura V. Insuficiencia respiratoria aguda como forma de debut de síndrome de LambertEaton asociado a carcinoma pulmonar de células pequeñas. An Med Interna (Madrid) 2002; 19: 243-245.

\section{INTRODUCCIÓN}

Lambert y cols. (1) describieron en 1961 un síndrome miasténico asociado a neoplasias, incluyendo diversas características electromiográficas típicas en un grupo de pacientes, la mayoría de los cuales tenían asociado carcinoma pulmonar de células pequeñas. Se caracteriza por la tríada: debilidad fluctuante de predominio en musculatura bulbar y proximal de miembros inferiores, reducción o abolición de los reflejos osteotendinosos (que característicamente reaparecen tras un periodo de contracción muscular sostenido) y alteraciones autonómicas colinérgicas (estreñimiento, sequedad bucal y ocular, impotencia y alte- raciones pupilares). El diagnóstico es electromiográfico, siendo característicos, la disminución de amplitud del potencial de motor evocado, la respuesta decremental a bajas frecuencias y la respuesta incremental o potenciación con altas frecuencias o tras una contracción maximal (2). Sin embargo, clínica y electromiográficamente puede solaparse con la Miastenia gravis, existiendo casos de presentación simultánea (3).

Por ello, el diagnóstico de confirmación se realiza mediante la detección de anticuerpos específicos contra los canales de calcio voltaje dependientes tipo $\mathrm{P} / \mathrm{Q}$, a diferencia de la miastenia gravis donde se detectan los anticuerpos antireceptores de acetilcolina (4).

Trabajo aceptado: 4 de noviembre de 2000

Correspondencia: Francisco Jover Díaz. C/ Amadeo de Saboya 2, 5B. 03012 Alicante. 
Presentamos el caso de un paciente afecto de insuficiencia respiratoria aguda (IRA) como forma de debut de un carcinoma microcítico de pulmón secundario a un síndrome de Lambert-Eaton (SLE). Dicha manifestación es excepcional y en nuestro conocimiento es el primer caso publicado en la literatura española.

\section{CASO APORTADO}

Varón de 53 años, fumador de 20 cigarrillos/día y bebedor de 40 gramos de alcohol al día con antecedentes de pleuritis a los 14 años. Consulta por disnea de reposo, obnubilación y cianosis de una semana de evolución. Refería un cuadro de 4 meses de evolución de fatigabilidad, disfonía, ptosis con visión doble bilateral e incapacidad para la deambulación, así como tos seca y pérdida de 10 kilos de peso. El paciente estaba taquipneico con incoordinación toracoabdominal, mostraba ptosis bilateral, pérdida de motilidad en cintura escapular, musculatura cervical y en todas las extremidades con predominio distal, así como arreflexia en ambos miembros inferiores e hiperreflexia de miembros superiores, siendo el resto de la exploración normal. La gasometría arterial respirando aire ambiente mostró: $\mathrm{pH} 7,15, \mathrm{pO}_{2} 45$, $\mathrm{pCO}_{2} 97, \mathrm{HCO}_{3}^{-} 34$ y la analítica general incluyendo hemograma, bioquímica, función renal y hepática fue normal. Precisó intubación y ventilación mecánica inmediata, ingresando en UCI. Una radiografía de tórax mostró una masa parahiliar derecha junto con paquipleuritis residual. Fibrobroncoscopia: compresión extrínseca en los segmentos S1 y S3 del bronquio del LSD. La TAC reveló la presencia de una masa de $3 \mathrm{~cm}$ en LSD próxima al hilio derecho y una masa mediastínica que provocaba el desplazamiento de la vena subclavia derecha. La biopsia transbronquial mostró infiltración por carcinoma de células pequeñas. Se realizó una TAC craneal, gammagrafía ósea y eco abdominal como estudio de extensión que resultó negativo. El estudio electrofisiológico mostró una disminución de amplitud de los potenciales sensitivos de los nervios mediano y ciático poplíteo izquierdos, apareciendo signos de denervación severa de los grupos musculares de las extremidades inferiores con signos de atrofia. Se realizó una estimulación repetitiva del nervio cubital izquierdo mostrando una alta facilitación (700\%) tras estimulación a altas frecuencias con un potencial motor basal de muy baja amplitud. Todos estos hallazgos fueron compatibles con una polineuropatía sensitivo-motora de tipo axonal de predominio en extremidades inferiores. El paciente permaneció consciente aunque sin ventilación espontánea, fracasando todos los intentos de desconexión del respirador mecánico por hipoventilación severa, falleciendo el quinto día del ingreso.

\section{DISCUSIÓN}

El síndrome de Lambert-Eaton (SLE) es un trastorno raro de la transmisión neuromuscular en el cual existen autoanticuerpos dirigidos directamente contra los canales de calcio voltaje-dependientes de tipo P/Q (omega-conotoxin MVIIC) localizados en el terminal presináptico del nervio. Estudios recientes $(5,6)$ han identificado dos epítopes (regiones S5-S6) de la sinaptotagmina (una proteína "ligadora" de calcio y fosfolípidos integrada en las membranas de las vesículas sinápticas), como potenciales antígenos contra los que actuarían los anticuerpos. En el caso del SLE asociado a carcinoma pulmonar de células pequeñas, los autoanticuerpos dirigidos contra los determinantes antigénicos de los referidos canales de calcio de las células tumorales actuarían de forma cruzada contra el receptor de la terminal presináptica. Se trata de un proceso que con frecuencia se asocia a tumores y en muchos casos su aparición antecede a la del tumor en meses.
Se ha descrito su asociación con todos los tipos de carcinoma pulmonar, pero predominantemente al carcinoma pulmonar de células pequeñas. Su frecuencia de aparición es baja y en este sentido en una serie de 641 pacientes con carcinoma pulmonar de células pequeñas, el 30\% tuvieron al menos una alteración neurológica en el momento del diagnóstico o durante su evolución clínica, predominando las metástasis cerebrales $(76 \%)$, siendo la incidencia de carcinoma pulmonar de células pequeñas sólo del 1\%(7). En otras series su incidencia llega hasta el 3\% (8). Se ha asociado a otros tipos de cáncer como linfomas, próstata, laringe, mama, digestivos o renal, así como a procesos autoinmunes concomitantes (9).

La presentación de IRA como forma de debut de carcinoma pulmonar de células pequeñas es excepcional, aunque son escasos los estudios que han investigado la afectación de la musculatura respiratoria en pacientes con SLE, pudiendo estar su frecuencia infravalorada debido a la ausencia de síntomas que orienten hacia su existencia. Hemos hecho una revisión mediante MEDLINE 1966-Diciembre 1999 encontrando sólo 19 casos descritos en la literatura. Gracey y cols. (10) describen los 5 primeros casos de desarrollo espontáneo de IRA en pacientes que precisaron ventilación mecánica. En todos se confirmó el diagnóstico de carcinoma pulmonar de células pequeñas y se demostró la existencia de SLE por electromiografía. En 1996, Gordon y cols. (11) revisaron los 12 casos descritos hasta la fecha, de los cuales 9 habían sido diagnosticados de carcinoma pulmonar de células pequeñas. Ninguno sobrevivió mas de un año tras el diagnóstico.

Existen muy pocos datos referentes a la afectación de la musculatura del sistema respiratorio en el SLE, aunque se ha comprobado la afectación de la musculatura respiratoria en casos de SLE incluso en ausencia de clínica, lo que demuestra la importancia de la realización de estudios electromiográficos para el diagnóstico correcto de este tipo de afectación. Se han descrito diversas características electromiográficas que permitirían el diagnóstico precoz de la debilidad de los músculos inspiratorios y en concreto del diafragma. El descenso marcado de la presión inspiratoria máxima (PI max), que aumenta tras la repetición de maniobras inspiratorias máximas, así como, la facilitación del potencial motor evocado mediante estimulación frénica repetitiva a altas frecuencias se consideran diagnósticas de la afectación diafragmática del SLE (12). Otro estudio, mostró la marcada disminución del potencial motor evocado con estimulación a frecuencias normales, y una respuesta decremental a bajas frecuencias $(3 \mathrm{~Hz})$, así como la potenciación de la respuesta $(>100 \%)$ con frecuencias altas $(40 \mathrm{~Hz})$, hallazgos típicos del SLE(13). La verdadera evaluación de la afectación de la musculatura respiratoria en pacientes con SLE sobre todo en las etapas precoces puede realizarse con la medición de las presiones máximas respiratorias (11). La aparición de nuevas técnicas electrofisiológicas aplicadas al sistema respiratorio podrían ayudar a la mejor identificación de esta afectación, entre ellas, la ausencia de respuesta del nervio frénico con el aumento de la actividad espontánea del diafragma, secundaria a la denervación del mismo de forma "funcional" por el bloqueo de la transmisión neuromuscular (12).

El tratamiento del SLE se ha enfocado desde dos puntos de vista: a) etiológico o modificador de la enfermedad y b) sintomático. En el primero se han utilizado corticoides, inmunosupresores, plasmaféresis e inmunoglobulinas intravenosas. Yuste y cols. (14), utilizando ciclosporina-A ( $3 \mathrm{mg} / \mathrm{kg} / \mathrm{día})$ 
consiguieron una completa recuperación clínica y electrofisiológica en una paciente tras cuatro meses de tratamiento. Newsom-Davis (15) utilizaron plasmaféresis durante un periodo de 20 días en pacientes con SLE, mostrando una mejoría clínica y electromiográfica lenta (10-20 días), que sin embargo desapareció en 3 semanas. Lang y cols. (16) usaron preparaciones de IgG de pacientes con SLE encontrando mejor respuesta que con plasma sólo, infiriendo los autores que la fracción de IgG contenía factores activos frente a los anticuerpos. El tratamiento de la neoplasia de base se ha comprobado como una medida eficaz. En cuanto al tratamiento sintomático, se han utilizado inhibidores de la acetilcolinesterasa que disminuyen la degradación de la acetilcolina mejorando así la transmisión neuromuscular. La guanidina ha ofrecido algunos resultados positivos (10), pero su intolerancia renal y hematológica limitan su uso. Las aminopiridinas (4-aminopiridina y 3,4 diaminopiridina) son otros agentes disponibles que bloquean los canales de $\mathrm{K}+\mathrm{y}$ aumentan el potencial de acción y la liberación de acetilcolina. La 3,4-diaminopiridina (3,4DAP) parece liberar más acetilcolina en la unión neuromuscular habiendo estudios que han demostrado su eficacia y seguridad $(9,17)$. Mc Evoy y cols. (18) plantearon un estudio prospectivo, doble ciego controlado con placebo con administración por vía oral de 3,4-DAP (15-20 mg cada 6 horas) en 12 pacientes con SLE, de los cuales 7 tenían carcinoma pulmonar de células pequeñas asociado, encontrando mejoría subjetiva y de las manifestaciones disautonómicas de forma persistente y con escasos efectos secundarios en todos los pacientes, así como mejoría de los parámetros de función muscular relacionada con la dosis administrada. Por tanto, el 3,4-DAP parece un agente prometedor en el tratamiento a corto y largo plazo del SLE, que junto con la adición de piridostigmina y fármacos inmunosupresores, podrían potenciar su efecto beneficioso.

Concluimos que el síndrome de Lambert-Eaton es una causa rara de IRA de origen neuromuscular; no obstante, la afectación respiratoria en el SLE puede ser una forma de expresión de la enfermedad más frecuente de lo que hasta ahora se ha reconocido, debido quizás a factores de confusión coexistentes (patología pulmonar previa, fármacos), debiendo descartarse en todo paciente con IRA de origen no filiado, sobre todo si se sospecha una patología neoplásica de base. La identificación de la debilidad musculatura respiratoria asociada al SLE es de gran importancia para un enfoque terapéutico correcto y por tanto, la realización de estudios electromiográficos de la musculatura respiratoria y en concreto del diafragma puede ayudar a la identificación de este tipo de afectación e instaurar un tratamiento precoz. A pesar de que el tratamiento de la neoplasia de base o la utilización de fármacos inmunosupresores sean medidas terapéuticas útiles, lo son a largo plazo y por lo tanto el uso de fármacos como el 3,4-DAP puede ser de elección por su acción inmediata y escasos efectos secundarios. Finalmente, debe descartarse la afectación de la musculatura respiratoria en pacientes con SLE, sobre todo si asocian carcinoma pulmonar de células pequeñas, por la posibilidad de un fallo respiratorio agudo, valorando la utilización precoz de fármacos que mejoren la transmisión neuromuscular.

\section{Bibliografía}

1. Lambert EH, Rooke ED, Eaton LM, Hodgson CH. Myasthenic syndrome occasionally associated with bronchial neoplasm: neurophysiologic studies. In: Viets HR, ed. Proceedings of Second International Symposium on Myasthenia Gravis. Springfield, Illinois. Charles C Thomas. Publisher, 1961: 362-410.

2. O'Neill JH, Murray NMF, Newsom-Davis J. The Lambert-Eaton myasthenic syndrome. A review of 50 cases. Brain 1988;111:577-596.

3. Newsom-Davis J, Leys K, Vincent A, Ferguson Y, Modi G, Mills K. Immunological evidence of the co-existence of the Lambert-Eaton myasthenic syndrome and myasthenia gravis in two patients. J Neurol Neurosurg Psychiatry 1991; 54: 452-53.

4. Vanda AL, Kryzer TJ, Griesmann GE, O'Suillebian PE, Windebank AJ, Woppmann A et al. Calcium-channel antibodies in the Lambert-Eaton Syndrome and other paraneoplastic syndromes. N Engl J Med 1995; 332: 1467-74.

5. Takamori M. An autoimmune channelopaty associated with cancer: Lambert-Eaton myasthenic syndrome. Intern Med 1999; 38 : 86-96.

6. Nakao YK, Motomura M, Suenaga A, Nakamura T, Yoshimura T, Tsujihata $\mathrm{M}$ et al. Specificity of omega-conotoxin MVIIC-binding and blocking calcium channel antibodies in Lambert-Eaton myasthenic syndrome. J Neurol 1999; 246 :38-44.

7. Sculier JP, Feld R, Evans WK, DeBoer G, Shepherd FA, Payne DG et al. Neurologic disorders in patients with small cell lung cancer. Cancer $1987 ; 60: 2275-83$.

8. Erlintong GM, Murray NMF, Spiro SG, Newsom-Davis J. Neurological paraneoplastic syndromes in patients with small cell lung cancer. A prospective survey of 150 patients. J Neurol Neurosurg Psychiatry $1991 ; 54: 764-767$

9. Murray NMF, Newsom-Davis J, Karmi Y, Wiles CM. Oral 3,4 Diaminopyridine in the treatment of the Eaton-Lambert myasthenia syndrome (LEMS). J Neurol Neurosurg Psychiatry 1984; 47 : 1052-3.

10. Gracey DR, Southorn PA. Respiratory Failure in Lambert-Eaton Myast henic Syndrome. Chest 1987; 91: 716-19.

11. Gordon-Smith A, Wald J. Acute ventilatory failure in Lambert-Eaton Myasthenic Syndrome and its response to 3,4-diaminopyridine. Neurology 1996:46: 1143-1145.

12. Wilcox PG, Morrison NJ, Anzarut AR, Pardy RL. Lambert-Eaton Myasthenic Syndrome involving the diaphragm. Chest 1988; 93: 604-606.

13. Nicolle MW, Phil D, Steward DJ, Remtulla H, Chen R, Bolton CF Lambert-Eaton Myasthenic Syndrome presenting with severe respiratory failure. Muscle Nerve 1996; 19: 1328-1333.

14. Yuste Ara JR, Beloqui Ruiz O, Artieda González-Granda J, Herrero Santos JI, De la Peña Fernández A, Prieto Valtueña J. Ciclosporina-A como tratamiento del síndrome miasteniforme de Eaton-Lambert. An Med Interna (Madrid) 1996; 13: 25-26.

15. Newsom-Davis J, Murray N, Wray D, Prior C, Gilvit M et al. LambertEaton Myasthenic Syndrome: electrophysilogical evidence for a humoral factor. Muscle Nerve 1982; 5 (suppl 9): 17-20.

16. Lang B, Newsom-Davis J, Wray D, Vincent A, Murray N. Autoimmune aetiology for myasthenic (Lambert-Eaton) Syndrome. Lancet 1981; 2: 224-26.

17. Lundh H, Nilsson O, Rosén I. Treatment of Lambert-Eaton syndrome: 3,4-diaminopyridine and pyridostigmine. Neurology 1984; 34: 1324-30.

18. McEvoy K, Windeback A, Daube JR, Loe PA. 3,4 diaminopyridine in the treatment of Lambert-Eaton Myasthenic Syndrome. N Engl J Med 1989; 321: 1567-71. 